\title{
Norois
}

Environnement, aménagement, société

197 | 2005/4

Varia

\section{La composition végétale des haies bocagères est- elle liée à l'utilité agricole des plantes ligneuses ?}

Effects of changes in the agricultural practices on tree and shrub species composition of hedgerows

\section{Samuel Périchon}

\section{OpenEdition}

\section{Journals}

Édition électronique

URL : http://journals.openedition.org/norois/325

DOI : 10.4000/norois.325

ISBN : 978-2-7535-1545-1

ISSN : $1760-8546$

\section{Éditeur}

Presses universitaires de Rennes

\section{Édition imprimée}

Date de publication : 1 décembre 2005

Pagination : 69-81

ISBN : 978-2-7535-0238-3

ISSN : 0029-182X

\section{Référence électronique}

Samuel Périchon, « La composition végétale des haies bocagères est-elle liée à l'utilité agricole des plantes ligneuses ? », Norois [En ligne], 197 | 2005/4, mis en ligne le 15 décembre 2008, consulté le 19 avril 2019. URL : http://journals.openedition.org/norois/325; DOI : 10.4000/norois.325

Ce document a été généré automatiquement le 19 avril 2019

(c) Tous droits réservés 


\title{
La composition végétale des haies bocagères est-elle liée à l'utilité agricole des plantes ligneuses?
}

\author{
Effects of changes in the agricultural practices on tree and shrub species \\ composition of hedgerows
}

Samuel Périchon

\section{NOTE DE L'ÉDITEUR}

Cet article a été reçu le 23 février 2005 et définitivement accepté le 29 septembre 2005.

1 Le «bocage» est un terme scientifique utilisé en France pour désigner un paysage caractérisé par la présence de réseaux de structures linéaires de végétaux ligneux (Baudry et Jouin, 2003). L'absence de termes équivalents dans les autres langues ne signifie pas, loin s'en faut, que ce paysage soit une originalité des campagnes françaises. Dans la plupart des régions du monde, nous en retrouvons des formes plus ou moins abouties (Baudry et Perichon, 2005).

2 La finalité d'un maillage de haies reste difficile à établir tant les boisements linéaires concrétisent des projets variés: matérialisation des limites de propriété, barrières défensives, production de bois, brise-vent, lutte contre l'érosion, assainissement des terres insalubres, etc. Cela dit, leur aspect trahit habituellement les fonctions qu'on leur attribue. En Ille-et-Vilaine, les haies de futaie de chênes (Quercus pedunculata Ehrh., Quercus sessiliflora Salisb.), de hêtres (Fagus sylvatica Linné1), de châtaigniers (Castanea sativa Miller) ou de cerisiers sauvages (Prunus avium L.) sont destinées à la production de bois d'œuvre; les arbustes et les arbrisseaux épineux tels le prunellier sauvage (Prunus spinosa L.), les aubépines (Cratcegus monogyna Jacq., Cratcegus oxyacantha Jacq.) ou l'ajonc ( Ulex europaeus L.) sont employés pour édifier des haies défensives; les arbres à feuillage marcescent (Carpinus betulus L., Fagus sylvatica L.) lesquels sont parfois associés à des 
plantes buissonnantes (Corylus avellana L., Cornus sanguinea L., Sambucus nigra L., Prunus spinosa L.) forment l'essentiel des brise-vent, etc. Mais, qu'il s'agisse de produire du bois, de clore ou d'abriter un champ, il est quelque peu réducteur de catégoriser de la sorte les boisements linéaires, car l'évolution des pratiques agraires les détourne souvent vers d'autres objectifs que celui qui motivait leur installation (Luginbühl, 1995).

Les conditions du milieu constituent un élément tout aussi déterminant dans ce qu'expose le paysage végétal. En général, sous les climats tempérés, les haies à proximité des cours d'eau sont composées de saules (Salix cinerea L., Salix alba L., Salix viminalis L., Salix caprea L.), d'aulnes (Alnus glutinosa Gaertn.), de bouleaux (Betula pubescens Ehrh.), de frênes (Fraxinus excelsior L.) ou de trembles. Pour le hêtre, le noyer noir (Juglans nigra L.), l'érable sycomore (Acer pseudoplatanus L.), le châtaignier ou le noisetier, l'humidité est un facteur limitant. De même, l'acidité des terres influence inégalement la croissance des végétaux ligneux : elle stimule celle du bouleau verruqueux (Betula verrucosa Ehah.) ou du chêne rouge (Quercus borealis Michx.), mais gêne celle de l'aubépine monogyne. Enfin, s'opposent aux essences de lumière ou de pleine lumière comme l'aulne glutineux, le frêne, le tremble ou le chêne pédonculé, des essences d'ombre ou qui du moins s'en accommodent, comme l'aubépine épineuse ou le hêtre. Par conséquent, le processus de requalification évoqué précédemment doit composer avec des conditions naturelles particulières.

4 La présentation en France en 1976 de l'ouvrage de M. D. Hooper représente un événement majeur dans l'étude des paysages de haies. Longtemps par facilité ou par ignorance, on a cru que les plantes situées autour des champs étaient uniquement là en raison de la qualité des sols, des conditions météorologiques et de la fantaisie de ceux qui les avaient plantées. Dans la plupart des travaux scientifiques, les haies étaient considérées comme une simple production des sociétés. Or, en établissant une corrélation troublante entre la diversité des espèces ligneuses et l'âge des structures, l'auteur a révélé leur caractère plus ou moins spontané. Quelques années plus tard, un autre chercheur, R. K. Sutton (1985), corrobore la thèse en assurant qu'une partie des haies du Middle West américain résulte de l'abandon momentané de l'entretien des bordures de champ (période de récession économique). Indépendamment de la volonté de ceux qui en ont la charge d'entretien, il s'avère que certains végétaux perturbent les logiques socio-économiques inhérentes à la production des haies.

Rendre compte des transformations de la matérialité des boisements linéaires appelle des investigations pluridisciplinaires. Les résultats présentés ci-après s'inscrivent dans ce cadre avec une attention, certes, plus marquée pour la géographie, la sociologie, l'histoire agricole contemporaine et l'écologie. Afin de mieux comprendre l'évolution des paysages bocagers, trente familles d'agriculteurs ont été rencontrées dans le département. De manière plus précise, il s'agissait d'établir un lien entre le moindre recours au bois dans les ménages agricoles et les modifications constatées dans la composition végétale des haies champêtres.

\section{Les sites d'étude et la méthodologie employée}

6 Les quatre sites retenus comme terrain d'étude (fig. 1) témoignent assez bien de la diversité des paysages campagnards en Ille-et-Vilaine. Pour partie, cela relève de l'inégal attachement des agriculteurs aux traditions agraires. En somme, en fixant une gestion 
originale de l'espace, le paysage réfléchit l'image de ce qu'ils souhaitent montrer d'euxmêmes (Perichon, 2005).

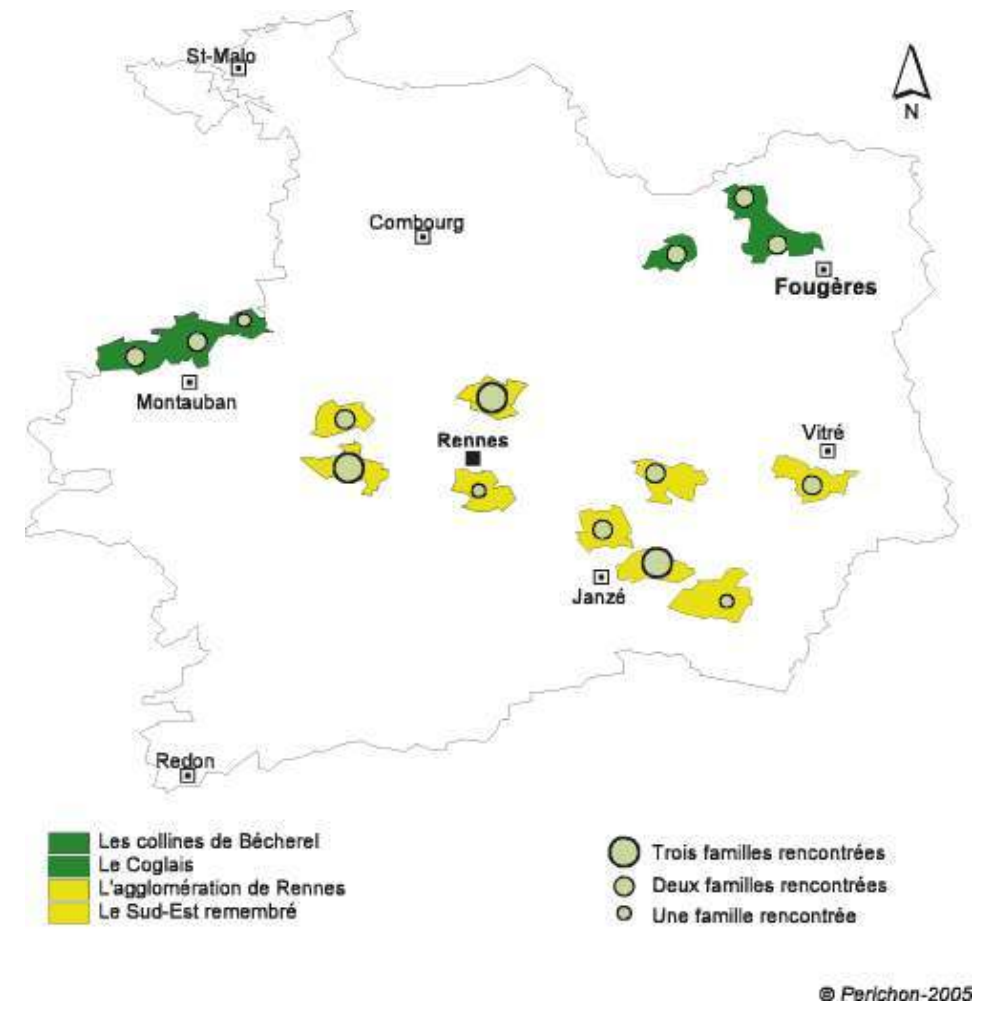

Figure 1 : Localisation des sites d'étude et répartition spatiale des familles rencontrées Localisation of research sites and spatial repartition of families of farmers

7 Le Coglais est une région située dans le nord-est du département, proche de Fougères et à environ $50 \mathrm{~km}$ de Rennes. Trois communes ont été étudiées : Saint-Marc-le-Blanc (1 228 ha et 761 habitants au recensement de 1999), Montours (1 556 ha et 825 hab.) et SaintGermain-en-Coglès ( 3265 ha et 1796 hab.). La Loisance, affluent du Couesnon dont la majeure partie du réseau hydrographique est tributaire, sépare l'espace défini (c'est-àdire le sud-ouest du Coglais) en deux plateaux. Le plus élevé, $160 \mathrm{~m}$ en moyenne, est disséqué par un réseau de vallées encaissées $(40$ à $60 \mathrm{~m}$ ) et toutes drainées. Le plateau inférieur (Saint-Marc-le-Blanc, sud de Saint-Germain-en-Coglès) incliné vers le sud-ouest, présente une altitude de l'ordre de $90 \mathrm{~m}$. Les deux ensembles forment les marges ouest du massif granitique de Fougères. Sans doute, est-ce afin de pallier les contraintes topographiques et géologiques que les populations habitantes ont naguère maillé le territoire de haies. Au regard de leur actuelle densité ( 90 à $120 \mathrm{~m} / \mathrm{ha}$ ), ces structures ont assurément conservé leur utilité pour fixer les terres agricoles sur les versants. Les reliefs agissent tout autant sur la mise en valeur des terres - les surfaces fourragères dont plus d'un quart sont des prairies naturelles, couvrent $81,6 \%$ de la surface agricole utile (SAU) laquelle influence les pratiques d'élevage: deux tiers des agriculteurs sont des producteurs de lait. En respect des réglementations européennes, la modeste superficie des exploitations (23,7 ha en moyenne) cantonne les cheptels à une trentaine de bovins. L'activité laitière est associée à une petite production avicole (36,7 \% des exploitations), plus rarement on engraisse des porcs (17,6\%). Depuis son classement en zone d'excédents structurels (ZES), la direction départementale de l'Agriculture et le conseil général s'intéressent de très près à l'évolution des pratiques agricoles dans cette sous-région. 
Leur vigilance se justifie d'autant plus que le Coglais fournit une part non-négligeable de l'eau potable consommée dans le département (agglomération de Rennes).

Médréac ( 2673 ha et 1517 hab.), Quédillac (2 673 ha et 962 hab.) et Saint-Pern (1 213 ha et 776 hab.) sont des communes limitrophes des Côtes-d'Armor, situés à mi-chemin entre Rennes et Saint-Brieuc. Malgré le passage de la route nationale à 4 voies reliant les deux villes, la région tend à se dépeupler. Entre 1962 et 1999, Quédillac a perdu $21,5 \%$ de ses habitants. La tendance s'inverserait depuis peu à Saint-Pern. Le paysage est fermé par un réseau bocager dense et ancien (autour $\mathrm{du} 90 \mathrm{~m} / \mathrm{ha}$ ) auquel s'ajoutent des haies nouvelles (5-10 m/ha). Notons une densité assez soutenue sur les interfluves (60 à $70 \mathrm{~m} / \mathrm{ha})$. Le relief ondulé (schistes peu ou pas cristallins) renforce l'impression de boisement, car il est difficile de prendre le recul nécessaire pour l'évaluer avec justesse. Curieusement sur le plan paysager l'agriculture s'éloigne de l'archétype des régions bocagères: prairies + bovins laitiers. Ceci se vérifie par le différentiel entre les surfaces en céréales ( $47,5 \%$ de la $\mathrm{SAU}$ ) et celles toujours en herbe (5,5\%), par la superficie moyenne des exploitations (supérieure de 10 ha à celle du département: $31,5 \mathrm{ha}$ ), et par le nombre d'unités pratiquant l'engraissement de porcs (38,6\%) ou de volailles (30,7 \%).

Avec la péri-urbanisation des campagnes, l'espace agricole autour de Rennes s'est réduit aux dépens du tissu bâti. La crainte de voir disparaitre un cadre recherché par la population habitante pour ses activités de plein-air, a contribué à ce que les élus reconsidèrent cet espace. Non-lieu aux portes de la ville, réserve foncière dont on peut disposer le jour venu, les terres cultivées sont depuis revendiquées par la société urbaine à la manière d'un lieu de nature accessible. Cet engouement se traduit, entre autres, par le développement du hobby-farming ${ }^{2}$. Près de $40 \%$ des exploitations de Betton $(2678$ ha et 8570 hab.), Saint-Gilles ( 2100 ha et 3507 ha), Mordelles (3 037 ha et 6013 hab.) et NoyalChâtillon-sur-Seiche (2 633 ha et 5600 hab.), sont tenues par des personnes ayant une autre activité professionnelle. Cela dit, la superficie concernée ne réunit que 12,7\% de la SAU. Les agriculteurs à plein temps se consacrent principalement à la production de lait. La moitié de la surface agricole utile est occupée par des fourrages (prairies naturelles : $8,9 \%$, prairies temporaires : $23,8 \%$, maïs fourrager : 17,4\%), le tiers par des céréales (blé tendre: $25,3 \%$, maïs grain : 7,4\%, orge : 2,6\%). Comme elle suscite des réactions négatives dans la communauté villageoise, l'activité hors sol est marginale : seules $11,2 \%$ des exploitations pratiquent l'engraissement de porcs. Le réseau de haies anciennes (40 à $50 \mathrm{~m} / \mathrm{ha}$ ) est également l'objet de pressions sociales. Le sachant fragile, les habitants pressent leurs élus pour qu'il soit protégé (classement au PLU) et/ou «rénové » (les nouvelles plantations représentent 20 à $30 \%$ du réseau).

Quatre des cinq communes sélectionnées dans le sud-est remembré, Chauméré (en Domagné : 2932 ha et 1642 hab.), Essé (2 346 ha et 868 hab.), Amanlis (2 561 ha et 1460 hab.) et Retiers (4 616 ha et 3554 hab.) se situent non loin de la D 41 reliant Rennes à Angers. Ajoutons Étrelles ( 795 ha et 2180 hab.), localité bordant le sud de Vitré. Trente à quarante kilomètres séparent cette grappe de communes du chef-lieu du département. Le relief mollement ondulé de la région (grès et schistes) a largement incité, dans les années cinquante et soixante, les agriculteurs à organiser de façon plus ou moins officielle des opérations de remembrement. Ces plans soutenus par les autorités ont conclu à la destruction totale des bocages. Depuis, rien n'a changé puisque l'examen des clichés de la mission photographique de 1996 indique une densité de haies inférieure à $20 \mathrm{~m} / \mathrm{ha}$ (Perichon, 2003). Conséquence ou non, l'agriculture est plus professionnalisée qu'ailleurs, les productions plus spécialisées et les agriculteurs plus jeunes. Fait majeur en Ille-et- 
Vilaine ( $1^{\mathrm{er}}$ producteur de lait en France), la part des exploitations vouées à la production de volailles ( $43,8 \%$ ) supplante celle des bovins laitiers (42,6\%). La capacité des élevages hors sol notamment avicoles, atteint des valeurs très supérieures à celles du département: 4351 unités de volailles/exploitation soit le quadruple de la moyenne départementale. La répartition des cultures et la superficie moyenne des exploitations (environ 30 ha) sont proches des valeurs enregistrées dans l'agglomération rennaise.

11 L'évolution des pratiques et des politiques agricoles, des usages domestiques et des habitudes de consommation ont singularisé le discours des trois dernières générations d'agriculteurs. Aussi, l'opportunité de confronter leurs points de vue a-t-elle été d'autant plus pertinente que le nombre de ceux qui ont vécu et participé à ces bouleversements historiques, diminue d'année en année. Le caractère sélectif de notre échantillon a causé quelques difficultés pour constituer une solide base de données. L'échantillon des familles interrogées s'est construit sans préférence d'activité ou de sexe quand bien sûr les épouses participaient à plein-temps à l'activité des exploitations.

Les entretiens ont été dirigés de façon individuelle pour éviter les pressions familiales. L'âge moyen des enquêtés est de 23,6 ans pour les enfants, 50,7 ans pour les parents et 78,2 ans pour les grands-parents. Les échanges enregistrés sur bande audio puis retranscrits duraient environ 40 minutes, les jeunes étant les moins inspirés (tableau 1). À partir de 19 photographies figurant par ordre de présentation: le marronnier (Aesculus hippocastanum L.), le bouleau verruqueux, le frêne, le noyer, l'aubépine épineuse, le châtaignier, le platane (Platanus x acerifolia Willd.), l'aulne glutineux, le noisetier, le cerisier sauvage, le charme, le sureau noir, le peuplier d'Italie, le chêne pédonculé, le prunellier sauvage, l'érable sycomore, le hêtre, le saule blanc et le tremble, les personnes interrogées devaient lister les produits et sous-produits associés. Il leur était également demandé de les localiser sur leur exploitation.

\begin{tabular}{|l|c|c|c|c|c|}
\hline & Homme & Couple & Femme & Âge moyen & Durée moyenne \\
\hline Enfants & 29 & 0 & 1 & 23,6 ans & $31,7 \mathrm{~min}$ \\
Parents & 18 & 12 & 0 & 50,7 ans & $44,2 \mathrm{~min}$ \\
Grands-parents & 5 & 16 & 9 & $78,2 \mathrm{ans}$ & $50,3 \mathrm{~min}$ \\
\hline Total & 52 & 28 & 10 & - & $42,1 \mathrm{~min}$ \\
\hline
\end{tabular}

Tableau 1 : Les statistiques des entretiens

Statistics of face-to-face interviews

\section{Les résultats}

Les résultats seront organisés en deux parties. Dans une première partie, nous dresserons (selon les générations et les sites d'étude) un bilan de l'évolution des diverses utilisations des plantes du bocage. Dans une seconde partie, nous décrirons leurs dynamiques spatiales.

\section{Les produits et sous-produits réalisés à partir des plantes des haies}

L'examen intergénérationnel a révélé trois tendances (fig. 2). 


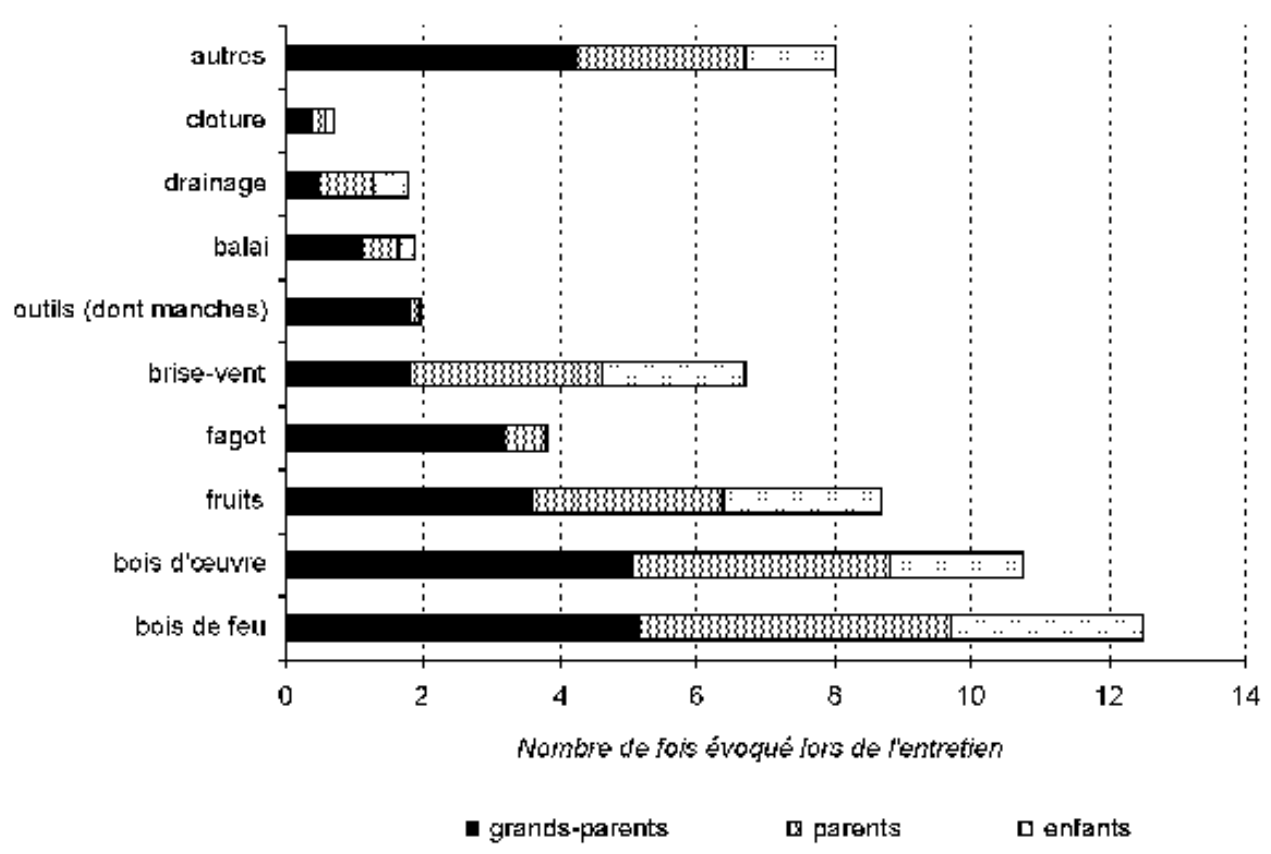

Figure 2 : Les produits et sous-produits réalisés à partir des plantes ligneuses des haies bocagères selon les générations

The main uses of trees and shrubs according to the generations

D'abord nous sommes bel et bien témoin de la disparition d'une quantité significative de produits et sous-produits réalisés à partir des plantes bocagères. Le meilleur exemple est sans doute celui du fagot. Pour la génération des 70 ans et plus, la taille des arbres d'émondes et l'entretien des taillis composaient l'essentiel des ressources en bois, car la plupart d'entre eux n'étaient pas propriétaires des terres qu'ils cultivaient. Autour de Rennes, le fagotage était une activité très rentable: le bois ainsi lié approvisionnait les boulangeries (cuisson du pain) et les laveries (faire bouillir l'eau). Désormais seuls $20 \%$ des agriculteurs fagotent une partie du produit des tailles de leurs haies... le reste est brûlé à même le champ. Le façonnage des manches d'outils, des cercles de tonneaux, des merlins, des balais, la fabrication de jouets (flûtes, sifflets, toupies, etc.), la préparation de boissons (liqueurs, limonades), toutes ces traditions populaires ont subi le même sort: disqualifiées, marginalisées, finalement oubliées.

Du fait du choix pour des énergies moins contraignantes (électricité, gaz), de l'accès à la propriété $^{3}, d u$ moindre coût des structures métalliques, produire du bois sur l'exploitation n'est plus indispensable. Nous l'avons bien ressenti dans les témoignages des enfants : le bois de chauffage est évoqué moins de trois fois, le bois d'œuvre moins de deux. À titre de comparaison, les grands-parents les citent à plus de cinq reprises. Reste à savoir si ce relâchement ne provient pas d'un recentrage vers quelques essences précieuses.

17 Signalons l'émergence de nouvelles fonctions comme le brise-vent, le drainage des terres ou le maintien des rives le long des cours d'eau. L'intégration de la haie dans un modèle scientifique agronomique sensibilise surtout les moins de 40 ans. Le brise-vent est la troisième fonction la plus souvent nommée par les parents et les enfants. Le gain entre la génération des enfants et celle des grands-parents tend cependant à se réduire $(+0,3$ point). 
Comme les propriétés intrinsèques des plantes déterminent les usages qui en sont faits, les espèces étudiées ont été affectées de manière inégale. Logiquement les essences ayant un bon comportement au feu, des fruits comestibles et/ou un bois résistant et facile à façonner ont mieux résisté que celles intervenant uniquement dans des productions d'outils, d'équipements, de jouets ou de boissons. Ceci n'exclut pas que le nombre moyen de produits et sous-produits associés soit dans les deux cas en recul : le chêne pédonculé est passé de 3,0 produits et sous-produits chez les grands-parents à 1,5 chez les enfants ; le châtaignier de 3,7 à 2,3; le noisetier de 2,5 à 1,4.

9 Pour le prunellier sauvage, l'aubépine épineuse, le sureau noir et le bouleau verruqueux, la situation est préoccupante. Jusqu'au début des années 1960, les deux épines par exemple étaient très employées : les baies de l'une entraient dans la composition d'une liqueur estimée $e^{4}$ leurs branches servaient à faire des balais ${ }^{5}$, des fagots ${ }^{6}$ parfois des outils. Plantées en haies ou dans les haies, elles permettaient de dresser des barrières infranchissables autour des parcelles de culture. Les grands-parents relèvent également leur intérêt écologique, leur valeur esthétique et symbolique (floraison annonciatrice de la fin de l'hiver). Une génération plus tard... on n'en retient que des contraintes d'entretien!

Dans le cadre du modèle Soltner (1988) ou de l'Institut pour le Développement Forestier (IDF, 1995 ; IDF, 1988; IDF, 1981), certains arbres ont profité, un temps, des politiques bocagères pour conforter leur place dans les pratiques agraires. Le charme, l'aulne glutineux et l'érable sycomore ont ainsi été recommandés aux agriculteurs désireux d'installer des brise-vent autour de leurs champs ou en périmètre des sièges d'exploitation (Luginbühl, 2003). Le feuillage marcescent du premier permettait en outre de cacher tout au long de l'année des bâtiments d'élevage peu esthétiques. Pour limiter les importations de bois en provenance des pays scandinaves, l'installation des peupleraies a tout autant été subventionnée par les pouvoirs publics.

Comme hier, les plantes d'agrément (marronnier, platane) sont négligées par les agriculteurs. Réserver un même sort au frêne et au cerisier sauvage est plus inattendu. Dans de nombreuses régions de France comme d'Europe, le frêne jouit en effet d'un réel intérêt auprès des populations agricoles (Bargioni et Sulli, 1998 ; Haeggström, 1998), cela est lié à sa capacité à assainir les terres humides, aux qualités nutritives de ses feuilles (fourrage pour les animaux d'élevage) et à son bon comportement au feu. Pareillement le cerisier sauvage est pourvu d'un vrai potentiel : bois d'œuvre, fruits, floraison printanière (esthétique), pratique de plaisance (cueillette des merises), etc. En l'état de nos recherches, il est difficile d'avancer des hypothèses pour rendre compte de la situation.

Parallèlement aux entretiens individuels, des questionnaires d'enquête ont été distribués aux agriculteurs en activité dans les communes concernées ou limitrophes ${ }^{7}$. Il en ressort de profondes divergences dans la manière dont chacun perçoit la haie en fonction de son lieu de résidence (tableau 2).

\begin{tabular}{|l|c|c|c|c|}
\hline & Le Coglais & $\begin{array}{c}\text { Les collines de } \\
\text { Bécherel }\end{array}$ & $\begin{array}{c}\text { L'agglomération de } \\
\text { Rennes }\end{array}$ & $\begin{array}{c}\text { Le Sud-Est } \\
\text { remembré }\end{array}$ \\
\hline Figurer les limites de propriété & $1,7 \%$ & 2,1 & 6,4 & 1,5 \\
Fixer les sols agricoles & 14 & 12,6 & 6,4 & 5,8 \\
Produire du bois & 18,2 & 16,5 & 7,7 & 7,4 \\
Abriter les animaux d'élevage & 34,9 & 25,1 & 20,5 & 5,9 \\
Abriter le siège d'exploitation & 8,7 & 17,3 & 21,8 & 16,2 \\
Embellir le territoire & 22,5 & 26,4 & 37,2 & 63,2 \\
\hline
\end{tabular}

Tableau 2 : Ce qu'attendent les agriculteurs d'une haie The functions of hedgerows 
Dans le Coglais et dans une moindre mesure autour de Bécherel, les boisements linéaires sont intégrés dans un modèle de développement agricole structuré autour de l'élevage de bovins laitiers en plein air. Les arbres et les arbustes pourvoient aussi aux besoins en bois de ceux qui en ont la charge d'entretien. Soulignons que le combustible est la base du chauffage domestique de plus d'un ménage agricole sur deux ${ }^{8}$ (contre 20,6 \% dans l'agglomération de Rennes et seulement 9,8\% dans le Sud-Est remembré). Ils constituent enfin des barrières naturelles efficaces contre l'érosion des sols agricoles.

Dans les campagnes peu pourvues d'arbres, les haies sont plutôt appréciées au travers de normes esthétiques. Pour 63,2 \% des enquêtés, c'est là leur principale fonction. À la faveur d'une forte demande sociale de paysage, cette requalification gagne les campagnes périurbaines.

25 Partout où l'agriculture se place sous le signe de la performance et du productivisme (Sud-Est remembré), les traditions (fagots, balais, jouets, boissons, etc.) sont disqualifiées. Des savoir-faire précis autour de plantes tels le bouleau verruqueux, le sureau noir, l'aubépine épineuse, le prunellier sauvage ou le charme, sont perdus (fig. 3). De même les fruits des champs: marrons et châtaignes, noisettes ou noix, ne sont plus récoltés (Perichon, 2004). Les prairies naturelles cloisonnées de haies vives de saules blancs, d'aulnes glutineux, de peupliers d'Italie sont remplacées par des champs ouverts ensemencés de maïs fourrager (Sud-Est remembré, collines de Bécherel, agglomération de Rennes). En l'absence de drains comme souvent c'est le cas dans le Coglais, l'assainissement des fonds de vallée reste assuré par des plantes ligneuses lesquelles conservent donc leur utilité agricole.

Aux dires des anciens, les enjeux sociaux autour de la composition végétale des haies ne sont pas chose nouvelle. Du temps de leurs parents et de leurs grands-parents, le choix des espèces cristallisait déjà des tensions dans les rapports à la terre. L'arbre des notables était un arbre au port majestueux, un arbre d'agrément ou de forêt, un hêtre, un platane ou un marronnier; à l'inverse, celui des colons était un arbre rustique, un arbre taillé pour ne pas trop pénaliser les cultures des parcelles, un arbre pourvoyeur de petit bois, un chêne dans l'agglomération de Rennes et dans les collines de Bécherel, un châtaignier dans le Coglais et le Sud-Est remembré. Pour signifier leur autorité, les premiers obligeaient quelquefois les seconds à planter autour des champs des essences de plaisance. En accédant à la propriété dans les années 1950, 1960, les anciens colons ont célébré l'instant en les arrachant des talus. Dans le Coglais, étrangement, beaucoup ont conservé ces arbres. De toute évidence, c'est là une autre stratégie d'affirmation sociale. S'agissant de l'utilité du frêne, du tremble, du noisetier et du cerisier sauvage, le contexte agricole n'aurait que peu d'influence. 


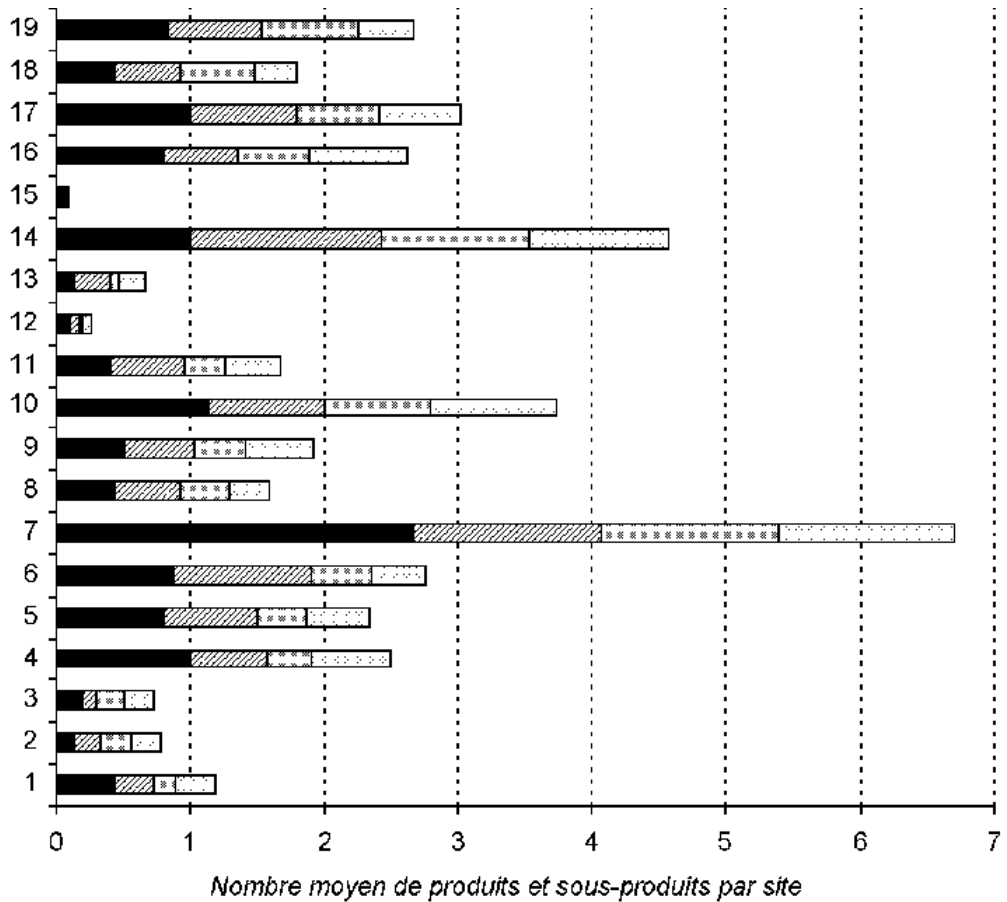
- Le Coglais
Les collines de Béchere
- L'agglomération de Rennes
$\square$ Le Sud-Est remembré

Figure 3 : Les produits et sous-produits réalisés à partir des plantes ligneuses des haies bocagères selon les sites d'étude

(1) Aulne glutineux; (2) Frêne ; (3) Tremble ; (4) Hêtre ; (5) Bouleau verruqueux ; (6) Prunellier sauvage ; (7) Châtaignier ; (8) Sureau noir ; (9) Aubépine épineuse ; (10) Noisetier ; (11) Charme ; (12)

Marronnier ; (13) Érable sycomore ; (14) Chêne pédonculé ; (15) Platane ; (16) Peuplier d'Italie ; (17) Noyer ; (18) Cerisier sauvage; (19) Saule blanc

The main uses of trees and shrubs according to the research sites 


\section{L'évolution des localisations préférentielles des plantes bocagères}

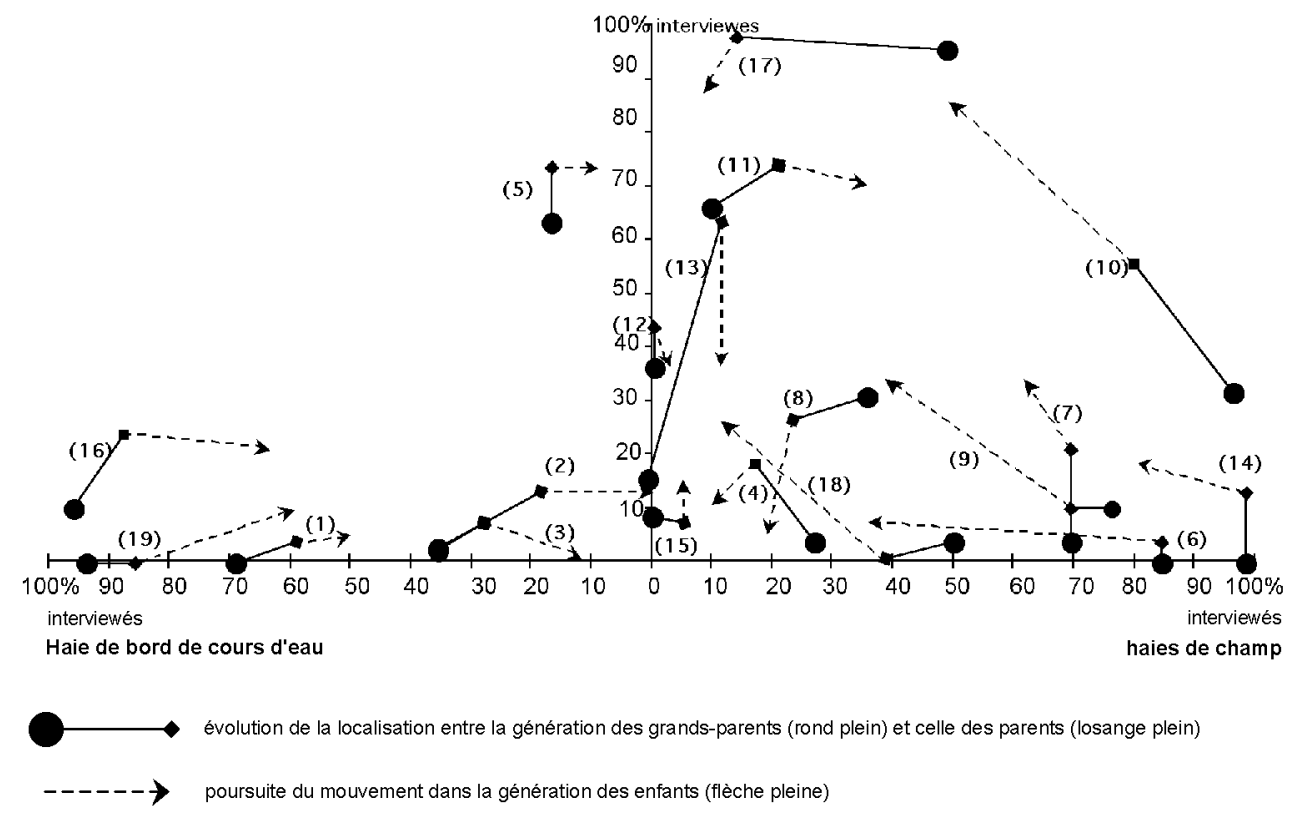

Figure 4 : L'évolution à travers trois générations d'agriculteurs des localisations préférentielles des plantes bocagères

(1) Aulne glutineux ; (2) Frêne ; (3) Tremble ; (4) Hêtre ; (5) Bouleau verruqueux ; (6) Prunellier sauvage ;

(7) Châtaignier ; (8) Sureau noir ; (9) Aubépine épineuse ; (10) Noisetier ; (11) Charme ; (12)

Marronnier ; (13) Érable sycomore ; (14) Chêne pédonculé ; (15) Platane ; (16) Peuplier d'Italie ; (17)

Noyer ; (18) Cerisier sauvage ; (19) Saule blanc

The localizations of plants according to three generations of farmers

Parce que l'espace agricole reste façonné par des logiques productives même si on parle de plus en plus de la valeur économique des aménités qu'il offre, les plantes qui y poussent, ne peuvent pas y échapper (fig. 4). Schématiquement, nous avons isolé :

Les plantes conservées en bordure de champ ou de ruisseau, et parfois installées en périmètre des sièges d'exploitation. Pour rendre compte de la situation (chêne pédonculé, châtaignier, peuplier d'Italie), les agriculteurs mettent en avant des aspects utilitaires, la production de bois en particulier. Leur projet d'organiser l'espace travaillé à ces fins se heurte toutefois à la capacité de certaines plantes (saule blanc, aulne glutineux) à coloniser et à croître dans des milieux contraignants. De sorte que s'oppose à une inertie voulue (bordures de champ), une inertie subie (abords de cours d'eau).

Les plantes écartées des haies champêtres et maintenues ou installées en périmètre des sièges d'exploitation. D'arbrisseau des champs, le noisetier est ainsi devenu une plante de jardin. Le différentiel intergénérationnel entre la localisation «dans les haies des champs » et "aux abords d'exploitation » l'atteste : +70 points pour les grands-parents contre -50 pour les enfants (soit un écart de 120 points). Pour le reste les variations sont de l'ordre de 45 à 60 points. Ces valeurs ne sauraient dissimuler des trajectoires contrastées. Le gain du noyer par exemple ne relève pas vraiment d'un redéploiement de l'arbre en périmètre des sièges d'exploitation puisque $95 \%$ des grands-parents le 
localisaient déjà en ces lieux. Le différentiel provient de deux processus indépendants : d'un côté l'arbre fruitier a été écarté des champs, de l'autre il a été conservé dans les jardins. La dynamique paysagère montre également ses limites avec le hêtre et le frêne : les enfants sont réticents à l'idée de les installer à proximité des bâtiments ou de l'habitation.

Le prunellier sauvage et le sureau noir sont les seules plantes ligneuses écartées des champs à ne pas avoir été replantées aux abords des fermes. Le premier étant et de loin celui qui a le plus pâti de l'évolution des pratiques agraires. Localisé «dans les haies des champs " par $85 \%$ des grands-parents et des parents, il ne l'est plus que par un tiers des enfants. Pour le second, la situation un peu différente puisque l'arbuste a d'abord été débarrassé des champs, puis exclu des jardins.

Exceptionnellement de nouvelles plantes investissent les haies champêtres. Pour le charme, nous enregistrons un gain de 25 points entre les grands-parents $(10 \%)$ et les enfants (35\%). Pour l'érable sycomore, l'évolution est plus «chaotique » et tient à l'influence des modes paysagères. Cela montre à quel point il est difficile pour une essence peu répandue de s'installer durablement autour des champs. La chose semble encore plus improbable pour le marronnier et le platane.

Pour les agriculteurs du Sud-Est remembré, aucune plante ne doit venir encombrer les bordures des champs. Si tel est le cas, ils l'interprètent comme le signe d'un jugement biaisé par la vision des fois archaïque des grands-parents. Différencions néanmoins les espèces détruites par leurs successeurs : l'aubépine épineuse, le bouleau verruqueux ou le noisetier; de celles, plus nombreuses, qui l'ont été par les parents: le saule blanc, le chêne pédonculé, le prunellier sauvage, l'aulne glutineux, le sureau noir, le charme et le peuplier d'Italie. Le second enseignement à retirer des investigations menées sur ce site tient au redéploiement massif des essences bocagères autour des sièges d'exploitation.

Dans l'agglomération de Rennes, si les agriculteurs cherchent eux aussi à se débarrasser du prunellier sauvage, de l'aubépine épineuse, du noisetier, du peuplier d'Italie et du saule blanc, dans le même temps, ils sont très attachés à ce que le chêne pédonculé et le châtaignier restent dans les champs. Le projet s'expliquerait tant par la valeur économique attribuée (bois de chauffage et bois de construction) que par les images suscitées: force, longévité, beauté, ruralité (Perichon, 2003). En outre, comme l'embellissement du cadre de vie procède de plantations au coup par coup d'essences exotiques, peu d'espèces locales (excepté le noisetier) y ont été installées.

Dans les autres bocages, les dynamiques spatiales sont contradictoires: maintien d'une localisation unique pour le chêne pédonculé, le châtaignier, l'aulne glutineux ou le saule blanc; double localisation pour le noisetier; exclusion des champs pour le cerisier et le prunellier sauvages; redéploiement vers les abords d'exploitation pour l'aubépine épineuse, le peuplier d'Italie et le sureau noir ; installation de nouvelles essences autour des champs (érable sycomore). Sur ce point, les agriculteurs du Coglais paraissent peu soucieux de distinguer les haies champêtres de celles des abords de ferme.

\section{Y a-t-il une corrélation entre la composition végétale des haies et l'utilité agricole des plantes bocagères ?}

La nature est bien évidemment un facteur perturbant dans la logique de production des haies. En dépit de l'opiniâtreté des agriculteurs de les faire disparaitre, certaines plantes 
apparaissent spontanément en bordure des parcelles. Simples rejets de souche ou graines transportées par les oiseaux, ces végétaux repoussent de printemps en printemps à la déception de ceux qui souhaitent les éloigner des champs. Ceci justifie un seuil de représentativité fixé à $74 \%{ }^{9}$ : le différentiel relèverait du boisement spontané.

Entre les générations, le coefficient de corrélation $\left(\mathrm{R}^{2}\right)$ varie de $56,5 \%$ à $72,2 \%$. Pour comprendre ces disparités, il est fondamental d'avoir en mémoire les bouleversements opérés dans les pratiques agraires depuis une cinquantaine d'années. Du temps des grands-parents, les haies étaient généralement fournies. La fonction défensive et la diversité des productions associées le justifiaient : à chaque plante correspondaient des usages précis. Au regard de leurs utilisations, l'aubépine épineuse, l'aulne glutineux, le charme, le frêne, le peuplier d'Italie, le saule blanc et le sureau noir étaient surreprésentés. Leur croissance végétale et leur capacité à coloniser les milieux contraignants (zones humides) en seraient à l'origine. D'autres, comme le hêtre, le châtaignier ou le bouleau verruqueux étaient sans doute en nombre insuffisant. Poursuivons notre examen par la génération des parents. Incontestablement, la moindre représentativité (56,5\% contre $72,2 \%$ chez les grands-parents) résulte des difficultés rencontrées pour se défaire des saules blancs, aulnes glutineux, prunelliers sauvages, aubépines épineuses, sureaux noirs voire des noisetiers. Paradoxalement, le nombre de plantes sous-représentées s'accroit, il concerne dorénavant le noyer, le hêtre, le charme, le bouleau verruqueux et le châtaignier. Conséquence de la recomposition des boisements linéaires engagée par les parents, le lien établi précédemment entre les plantes des haies et leurs utilisations agricoles se retrouve dans la génération des enfants ( $71,2 \%)$.

$\mathrm{Si}$ les logiques productives influencent la manière dont les agriculteurs des bocages gèrent leurs boisements $\left(\mathrm{R}^{2}=68,0 \%\right.$ dans le Coglais et les collines de Bécherel, 64,2 \% dans l'agglomération de Rennes), il n'en est plus de même dans les campagnes remembrées du sud-est du département $\left(\mathrm{R}^{2}=35,9 \%\right)$. Dans le cas présent, les arbres, les arbustes et les arbrisseaux sont clairement ressentis comme une contrainte : coût économique, charge de travail, nuisance aux cultures, etc. Leur densité autour des champs serait inversement proportionnelle au dynamisme économique des régions considérées. Par conséquent, seuls les végétaux les plus difficiles à se débarrasser (saule blanc, sureau noir et prunellier sauvage) sont momentanément lisibles autour des terres labourables. À l'inverse, dans les campagnes restées boisées et assez isolées, les agriculteurs ont tendance à conserver des plantes peu utiles. C'est surtout vrai pour l'aubépine épineuse, le prunellier sauvage et le sureau noir. Les enquêtés font preuve ici d'une réelle maturité en intégrant l'intérêt des végétaux dans un cadre plus vaste qu'une simple production agricole (écologie du paysage, notamment). De ce fait la représentativité est moins élevée qu'autour de Rennes, autre territoire bocager.

\section{Conclusion}

38 La plupart des productions traditionnelles en lien avec les plantes ligneuses des bocages ont disparu des habitudes des agriculteurs ou bien disparaissent sous nos yeux. Selon le lieu de résidence, le processus est plus ou moins abouti. Le désir récent de la société urbaine de renouer avec son passé agricole (chauffage au bois, récolte des châtaignes, etc. ) crée parfois des conflits entre les générations : les jeunes n'y voient qu'un effet de mode, leurs aînés, peut-être des débouchés économiques. Dans le Sud-Est remembré, la situation est moins problématique puisque tous ont fait le deuil de ces traditions. Enfin, dans les 
bocages campagnards, le recours à l'arbre cache souvent des préoccupations pécuniaires : économies d'électricité ou de gaz, repas peu coûteux pour les châtaignes, etc.

L'évolution des pratiques agraires autour des plantes a profondément modifié le paysage végétal du département. En perdant tout ou partie de leur intérêt agricole, nombre d'essences ont été écartées des bordures de champ puis, au gré des normes esthétiques établies, réinstallées non loin des bâtiments d'élevage ou de l'habitation. Mais quelle que soit la détermination des sociétés d'organiser leur environnement en fonction de concepts idéologiques spécifiques, nous ne devons pas perdre de vue que la nature est également créatrice de paysages.

La singularité des dynamiques paysagères et la diversité des paysages arborés qui en résulte aujourd'hui en Ille-et-Vilaine, montrent en outre combien la construction d'un territoire relève de confrontations permanentes de projets d'acteurs et de groupes sociaux différents sur des espaces eux-mêmes différents. En les matérialisant, les motifs des paysages agricoles visibles deviennent symptomatiques de situations socioéconomiques particulières. Dans les bocages, ce sont les haies qui jouent ce rôle d'information. Aussi, dès l'instant que nous cessons de les voir comme de simples objets de nature, que nous nous efforçons de comprendre les raisons de leurs formes si variables d'une région à l'autre, nous ne pouvons qu'être interpellés par ce qu'elles suggèrent à ceux qui les regardent ou les façonnent. Tout laisse penser en effet que derrière les espèces ligneuses privilégiées par les agriculteurs, la manière dont ils gèrent leurs boisements, le fait de les accepter ou au contraire de les exclure de leurs champs, peuvent être compris comme autant de réponses faites aux changements de la société dans laquelle ils vivent.

\section{BIBLIOGRAPHIE}

BARGIONI (E.), SULLI (A. Z.), 1998. - « The production of fodder trees in Valdagno, Vicenza, Italy », dans KIRBY (K. J.), WATKINS (C.) (ed.), The ecological history of european forests, Wallingford, p. 43-52.

BAUDRY (J.), JOUIN (A.), (coord.), 2003. - De la haie aux bocages - organisation, dynamique et gestion, INRA édition, $435 \mathrm{p}$.

BAUDRY (J.), PERICHON (S.), (à paraître). - « Les haies et les bocages dans le monde : éléments de comparaison ", bocages et sociétés, Université de Rennes 1/Université de Rennes 2.

HAEGGSTRÖM (C. A.), 1998. - « Pollard meadows: multiple use of human-made nature », dans KIRBY (K. J.), WALTKINS (C.), The ecological history of european forests, Wallingford, p. 33-42.

HOOPER (M. D.), 1976. - Études historiques et biologiques des haies anglaises, CNRS/INRA/ENSA/ Université de Rennes, p. 225-228.

INSTITUT POUR LE DÉVELOPPEMENT FORESTIER, 1995. - L'entretien courant des haies bocagères : les grands modèles d'entretien des haies, $68 \mathrm{p}$.

INSTITUT POUR LE DÉVELOPPEMENT FORESTIER, 1988. - « Les haies brise-vent : pour protéger, produire et aménager ", Forêt entreprise, 52, p. 9-68. 
INSTITUT POUR LE DÉVELOPPEMENT FORESTIER, 1981. - Cultiver les arbres feuillus pour récolter du bois de q ualité, $278 \mathrm{p}$.

LUGINBÜHL (Y.), 1995. - De l'utilité de l'émondage à la contemplation du paysage. CNRS/ENSP/INRA, 121

p.

-, 2003. - «Les politiques de reconstruction/protection du bocage et leurs effets ", Bocagement, reconstitution et protection du bocage, ministère de l'Environnement/INRA/CNRS, p. 21-66.

PÉRICHON (S.), 2003. - L'évolution des paysages d'arbres et de haies - histoire récente vue par trois générations d'agriculteurs, Thèse en sciences de l'environnement, Paris, ENGREF, 319 p.

-, 2004. - La tradition de la châtaigne dans les ménages agricoles d'Ille-et-Vilaine, Courrier de l'Environnement de l'INRA : [http://www.inra.fr/dpenv/pdf/perichc52.pdf].

-, 2005. - Les paysages campagnards sont-ils à l'image de ce que les agriculteurs souhaitent montrer d'eux-mêmes ?, L'Académie d'Agriculture de France : [http://www.academie-agriculture.fr/files/ publications/notes_recherche/200506note1.pdf].

SOLTNER (D.), 1988 (7éd.). - L'arbre et la haie, Sciences et techniques agricoles, 206 p.

SUTTON (R. K.), 1985. - « Relict rural planting in Eastern Nebraska », Landscape Journal, 4, p. 106-115.

\section{NOTES}

1. Linné est le seul auteur qui figure sous la seule initiale de son nom, L.

2. Agriculture pratiquée pendant le temps libre pour l'agrément.

3. La production de bois d'œuvre exige une gestion de l'arbre préjudiciable aux cultures.

4. Appelée «prunelle », cette boisson était autrefois élaborée à base d'eau-de-vie de pomme.

5. Les balais d'épines servaient à ramasser les feuilles mortes tombées de part et d'autre des talus, lesquelles étaient ensuite utilisées pour pailler les animaux d'élevage.

6. Employés pour cuire le pain ou ramoner les conduits de cheminées.

7. L'enquête a été menée auprès de 246 agriculteurs.

8. Les valeurs dépassent $90 \%$ quand on y ajoute le « chauffage d'appoint ».

9. Ce seuil correspond au coefficient de corrélation (R2) jamais dépassé dans notre échantillon (R2 maximum : grands-parents du bocage périurbain).

\section{RÉSUMÉS}

Depuis les années cinquante, le recours au bois s'est considérablement amoindri dans les ménages agricoles. Quantité de plantes bocagères ont alors perdu en partie, voire en totalité, leur attrait. Comme la production de haies s'inscrit dans des logiques économiques directes ou indirectes, il y a forcément eu modification de l'aspect des boisements. Avant de s'y consacrer, il est éclairant d'apprécier, à travers les témoignages de trois générations d'agriculteurs d'une même famille, combien les pratiques sociales autour des espèces des bordures de champ ont évolué dans le temps et l'espace. 
Because of technological progress, the recourse to wood has considerably reduced. The number of trees and shrubs have partly or even entirely lost their attraction. Just as inside the fields, the boundaries are connected directly or indirectly with economic reasoning. We are faced with a question: do the plants that become of no use still have their place around the fields? Before answering this question, it is interesting to appreciate, through face-to-face interviews with three generations of farmers from the same family, how much the socio-economic changes have affected the relationships of tree and shrub species in field boundaries.

INDEX

Keywords : agricultural practices, agriculture, hedgerow

Mots-clés : agriculture, haies, pratiques agricoles

Index géographique: France, Ille-et-Villaine

\section{AUTEUR}

\section{SAMUEL PÉRICHON}

RESO - UMR 6590 CNRS ESO, Université Rennes 2 Haute-Bretagne, Maison de la Recherche en Sciences Sociales (MRSS), Place du Recteur Henri Le Moal, 35043 Rennes cedex, France, samuel.perichon@uhb.fr 